\title{
Lower peak bone mass and abnormal trabecular and cortical microarchitecture in young men infected with HIV early in life
}

\author{
Michael T. Yin ${ }^{\mathrm{a}}$, Emily Lund ${ }^{\mathrm{a}}$, Jayesh Shah ${ }^{\mathrm{a}}$, Chiyuan A. Zhang ${ }^{\mathrm{a}}$, Marc Foca ${ }^{\mathrm{a}}$, Natalie Neu ${ }^{\mathrm{a}}$, \\ Kyle K. Nishiyama ${ }^{a}$, Bin Zhou ${ }^{b}$, Xiangdong E. Guo ${ }^{b}$, John Nelson, David L. Bella ${ }^{a}$ Elizabeth \\ Shane ${ }^{\mathrm{a}}$, and Stephen M. Arpadi ${ }^{\mathrm{a}}$ \\ aColumbia University Medical Center, Department of Biomedical Engineering, Columbia \\ University, New York, New York, USA \\ ${ }^{b}$ Bone Bioengineering Laboratory, Department of Biomedical Engineering, Columbia University, \\ New York, New York, USA
}

\begin{abstract}
Introduction-HIV infection and antiretroviral therapy (ART) early in life may interfere with acquisition of peak bone mass, thereby increasing fracture risk in adulthood.

Methods-We conducted a cross-sectional study of dual-energy X-ray absorptiometry (DXA) and high-resolution peripheral quantitative computed tomography (HR-pQCT) in $30 \mathrm{HIV}$-infected African-American or Hispanic Tanner stage 5 men aged 20-25 on ART (15 perinatally infected and 15 infected during adolescence) and 15 HIV-uninfected controls.
\end{abstract}

Results-HIV-infected men were similar in age and BMI, but were more likely to be AfricanAmerican $(P=0.01)$ than uninfected men. DXA-derived areal bone mineral density (aBMD) $Z$ scores were 0.4-1.2 lower in HIV-infected men at the spine, hip, and radius (all $P<0.05$ ). At the radius and tibia, total and trabecular volumetric BMD (vBMD), and cortical and trabecular thickness were between 6 and 19\% lower in HIV-infected than uninfected men $(P<0.05)$. HIVinfected men had dramatic deficiencies in plate-related parameters by individual trabeculae segmentation (ITS) analyses and 14-17\% lower bone stiffness by finite element analysis revealed. Differences in most HR-pQCT parameters remained significant after adjustment for race/ethnicity. No DXA or HR-pQCT parameters differed between men infected perinatally or during adolescence.

Conclusion-At an age by which young men have typically acquired peak bone mass, HIVinfected men on ART have lower BMD, markedly abnormal trabecular plate and cortical microarchitecture, and decreased whole bone stiffness, whether infected perinatally or during adolescence. Reduced bone strength in young adults infected with HIV early in life may place them at higher risk for fractures as they age.

(C) 2013 Wolters Kluwer Health | Lippincott Williams \& Wilkins

Correspondence to Michael T. Yin, MD, MS, Division of Infectious Diseases, Columbia University Medical Center, 630 West 168 th street, PH8-876, New York, NY 10032, USA. Tel: +1 212305 7185; fax: +1 212305 7290; Mty4@ columbia.edu.

Conflicts of interest

The other authors have no conflicts of interest. 


\section{Keywords}

bone microarchitecture; bone mineral density; bone strength; high-resolution peripheral quantitative computed tomography; peak bone mass; perinatal HIV infection

\section{Introduction}

Children who acquire HIV infection perinatally or in adolescence have the greatest cumulative exposure to potentially adverse effects of HIV infection and/or antiretroviral therapy (ART) on the skeleton. Peak bone mass is a key determinant of osteoporosis and fracture risk later in life [1]. If HIV infection and/or ART interfere with bone acquisition during childhood and adolescence resulting in lower peak bone size and mass, fracture risk may be increased in adulthood. Several cross-sectional studies have found both lower bone mineral content (BMC) and bone mineral density (BMD) by dual-energy $\mathrm{x}$-ray absorptiometry (DXA) in perinatally HIV-infected children compared with healthy children of similar age and sex [2-5], even after adjustment for stage of sexual maturation, height, and weight [2]. A recent study also found that behaviorally infected adolescent men on antiretrovirals had lower BMD by DXA than uninfected controls [6]. However, a major limitation of DXA is that it does not measure the anteroposterior diameter of bone. Therefore, measurement of areal BMD (aBMD) by DXA may underestimate true volumetric BMD (vBMD) especially in children with impaired growth, delayed pubertal development, and smaller bone size. Notably, the only published study comparing aBMD by DXA and vertebral vBMD by quantitative computed tomography (CT) in HIV-infected and uninfected children and adolescents found discrepant results [7]. No study has yet compared aBMD by DXA and vBMD by CT in HIV-infected and uninfected young adults after peak bone mass has likely been achieved. In addition, DXA cannot distinguish between cortical and trabecular bone, nor can it measure bone microarchitecture, which also influences fracture risk independent of aBMD [8]. High-resolution peripheral quantitative computed tomography (HR-pQCT) is a noninvasive imaging technology that provides a threedimensional measurement of true $\mathrm{vBMD}$ at the distal radius and tibia, and permits separate assessment of cortical and trabecular bone microarchitecture [9,10]. In addition, individual trabeculae segmentation (ITS)-based morphological analysis, a novel analysis technique for HR-pQCT images, can distinguish between trabecular plates, which play the dominant and critical role in mechanical competence of trabecular bone, from trabecular rods, and predict fracture status independently of aBMD [11-13].

We hypothesized that, compared with uninfected controls, individuals infected with HIVearly in life would have lower cortical and trabecular vBMD as well as abnormal trabecular and plate and rod microarchitecture, features that are associated with reduced bone strength and prevalent fractures in older men and women who are not infected with HIV [14]. Because perinatally infected men have had longer exposure to both the HIV virus and ART, we also hypothesized that vBMD would be lower and microarchitectural abnormalities more pronounced in perinatally infected men than those infected during adolescence. 


\section{Materials and methods}

\section{Study participants}

Between May and August of 2012, 45 (30 HIV-infected, 15 HIV-uninfected) AfricanAmerican or Hispanic men, aged 20-25 years and Tanner 5 developmental stage were recruited from the infectious diseases and general internal medicine clinics at Columbia University Medical Center (CUMC) in New York City. HIV-infected men were infected either perinatally $(n=15)$ or during adolescence $(n=15)$ and all were on ART. Exclusion criteria included history of fragility fracture or osteoporosis; metabolic bone disease; multiple myeloma or metastatic cancer; endocrinopathies; serum creatinine more than 1.5 $\mathrm{mg} / \mathrm{dl}$; liver, celiac or inflammatory bowel disease; and current glucocorticoid or anticonvulsant use. Fifteen HIV-uninfected controls meeting the same criteria with serostatus verified by enzyme-linked immunosorbent assay were also enrolled from an outpatient clinic within the same health system.

Medical, surgical, and sexual health history, including age of sexual debut, current tobacco and alcohol use, current and past medication history, HIV and ART history, current and nadir $\mathrm{CD} 4^{+}$T-cell counts, and HIV-1 plasma RNA levels were obtained by interview and chart review. Eligible participants self-identified their race/ethnicity and developmental stage was determined using self-assessment illustrations [15]. This study was approved by the Institutional Review Board of CUMC and all participants provided written informed consent.

\section{Bone density and body composition measurements}

aBMD of the lumbar spine (L1-4), femoral neck, total hip, nondominant $1 / 3$ radius, ultradistal radius (UDR), and body composition were measured by DXA on a QDR 4500 bone densitometer (Hologic Inc., Bedford, Massachusetts, USA) at CUMC. Short-term invivo precision is $0.68 \%$ for the lumbar spine, $1.36 \%$ for the total hip, and $0.70 \%$ for the radius. $Z$-scores, comparing aBMD to an age-matched, sex-matched, and race/ethnicitymatched reference population were derived for the hip from the National Health and Nutrition Examination Survey (NHANES III) and the manufacturer's normative database for the spine and forearm. Height $(\mathrm{cm})$ and weight $(\mathrm{kg})$ were measured by Harpenden stadiometer (Holtain Ltd., Crymych, UK) and balance beam scale, respectively. BMI was calculated as weight divided by squared height $\left(\mathrm{m}^{2}\right)$.

\section{High-resolution peripheral quantitative computed tomography measurements}

Cross-sectional area (CSA), vBMD, and microarchitecture were assessed at the nondominant distal radius and distal tibia by HR-pQCT (XtremeCT; Scanco Medical, Brüttisellen, Switzerland) as described in detail elsewhere [9,16,17]. Briefly, this system allows for high-resolution in-vivo evaluation of bone structure at a nominal isotropic resolution of $82 \mu \mathrm{m}$. The region of interest (ROI) consisted of an approximately $9 \mathrm{~mm}$ axial length of bone. At the radius, the ROI was located $9.5-18.5 \mathrm{~mm}$ proximal to the endplate. At the tibia, the ROI was located $22-31 \mathrm{~mm}$ proximal to the endplate [9,17]. Bone microarchitecture analysis was performed according to the manufacturers' standard evaluation protocol as previously described $[9,18,19]$. The following parameters were 
reported: CSA; total and trabecular bone density (total vBMD, Tb.vBMD; mg

hydroxyapatite $\left./ \mathrm{cm}^{3}\right)$; trabecular number $\left(\mathrm{Tb} . \mathrm{N} ; \mathrm{mm}^{-1}\right)$; thickness $(\mathrm{Tb} . \mathrm{Th} ; \mu \mathrm{m})$; and separation (Tb.Sp; $\mu \mathrm{m})$. The standard deviation of Tb.Sp (Tb.Sp.SD; $\mu \mathrm{m}$ ) was measured to assess the heterogeneity of the trabecular network.

\section{Cortical analyses}

To evaluate the cortical bone structure, a validated autosegmentation method [20,21] was applied to separate the cortical and trabecular compartments and measure cortical porosity (Ct.Po, \%), direct cortical thickness (Ct.Th, mm), and cortical BMD (Ct.BMD, mg hydroxyapatite $/ \mathrm{cm}^{3}$ ) [21,22]. Ct.Po is calculated as the amount of void space in the cortex using Image Processing Language (IPL, Version 5.08b; Scanco Medical). Ct.Th is measured directly using a distance transform method and Ct.BMD is defined as the average BMD in the cortical bone compartment $[20,21]$.

\section{Individual trabeculae segmentation analyses}

In addition to the standard analysis, ITS was applied to assess the orientation and the characteristics of plate and rod trabecular elements $[13,23]$. Briefly, this method creates a skeleton of the trabecular region and classifies each trabecular element as a surface or curve; through an iterative reconstruction method, each voxel of the original image is classified as belonging to either an individual plate or rod $[13,23]$. Based on the three-dimensional evaluations, plate and rod bone volume fraction (pBV/TV and $\mathrm{rBV} / \mathrm{TV}$ ) and plate and rod number (pTb.N and rTb.N, 1/mm) were evaluated. Plate-to-rod ratio (P-R ratio), a parameter of plate versus rod characteristics of trabecular bone, was defined as plate bone volume divided by rod bone volume. The average size of plates and rods was quantified by plate and rod thickness (pTb.Th and $\mathrm{rTb} . \mathrm{Th}, \mathrm{mm}$ ), plate surface area (pTb.S, $\mathrm{mm}^{2}$ ), and rod length (rTb.l, mm). Intactness of the trabecular network was characterized by plate-plate, platerod, and rod-rod junction density (P-P, P-R, and R-R Junc.D, $1 / \mathrm{mm}^{3}$ ), calculated as the total junctions between trabecular plates and rods normalized by the bulk volume.

Orientation of trabecular bone network was characterized by axial bone volume fraction (aBV/TV), defined as axially aligned bone volume divided by the bulk volume [13,23].

\section{Micro finite element analyses}

Each thresholded HR-pQCT whole bone image and trabecular bone compartment image of the distal radius and tibia was converted to a micro finite element $(\mu \mathrm{FE})$ model. Bone tissue was modeled as an isotropic, linear elastic material with a Young's modulus of $15 \mathrm{GPa}$ and a Poisson's ratio of 0.3 [18]. For each model, a uniaxial compression test was performed to calculate the reaction force under a displacement equal to $1 \%$ of the bone segment height along the axial direction. Whole bone stiffness, defined as reaction force divided by the applied displacement, characterizes the mechanical competence of both the cortical and trabecular compartments and is associated with whole bone strength [18] and fracture risk [19,24,25]. Similarly, trabecular bone stiffness characterizes the mechanical competence of trabecular bone compartment [18]. All the $\mu \mathrm{FE}$ analyses were performed using a customized element-by-element preconditioned conjugate gradient solver [26]. 


\section{Statistical analysis}

All statistical analyses were performed using SAS (version 9.2; SAS Institute, Cary, North

Carolina, USA). Continuous data are presented as mean value \pm standard standard deviation; categorical data are presented as percentage or absolute number. Means between groups were compared using Student's $t$-tests; covariate-adjusted means between groups were compared by analysis of covariance; between group differences in correlations between DXA and HR-pQCT measures were tested with analysis of variance test of homogeneity of slopes; categorical data compared by odds ratios from $\chi^{2}$, Fisher's exact, or JonckheereTerpstra test for trend. No adjustments were made for multiple comparisons. A $P$ value $<0.05$ was considered statistically significant.

\section{Results}

\section{Characteristics of the study population}

Compared with controls, HIV-infected men were similar in age and BMI, but were more likely to be African-American (Table 1). Perinatally infected men were on average 1 year younger than men infected during adolescence, but were similar with regard to BMI, race/ ethnicity, and $\mathrm{CD}^{+}{ }^{+}$cell counts (data not shown). Among perinatally infected men, mean current and nadir CD4 ${ }^{+}$cell counts were $474 \pm 161$ and $191 \pm 161$ cells/ $\mu$, respectively: $13 \%$ had a history of AIDS-defining illness, and the mean duration of ART was 12 years. Among men infected during adolescence, age of sexual debut was $15 \pm 3$ years, mean time since HIV diagnosis was $2.5 \pm 1.0$ years, and mean current/nadir CD4 ${ }^{+}$cell counts were 533 $\pm 157 / 299 \pm 149$ cells/ $\mu$ l; $6 \%$ had history of AIDS-defining illness and mean duration of ART was 2 years. Proportion with HIV-1 RNA levels less than 50 copies/ $\mu$ l was the same in each group (67 versus $67 \%, P=1.0)$. The majority of perinatally infected men $(67 \%)$ were on protease inhibitor-containing regimens, whereas the majority of men infected during adolescence (87\%) were on nonnucleoside reverse transcriptase inhibitor (NNRTI)containing regimens. Current tenofovir use was also higher in men infected during adolescence than perinatally infected men ( 93 versus $67 \%, P=0.07$ ), although this did not reach statistical significance.

\section{Dual-energy X-ray absorptiometry and bone turnover marker results}

Bone size, as assessed by CSA by DXA scans was similar among HIV-infected and HIVuninfected men at the spine, hip, and radius. However, aBMD Z-scores were 0.4-1.2 lower in HIV-infected men at all sites (Table 1). There were no significant differences in DXA measures between the two HIV-infected groups.

\section{High-resolution peripheral quantitative computed tomography and cortical porosity analyses}

At the radius, CSA was similar between groups, but total vBMD, trabecular vBMD, and cortical and trabecular thickness were between 6 and 19\% lower in HIV-infected than uninfected groups (Figs. 1 and 2). After adjusting for race/ethnicity, total and trabecular vBMD as well as cortical and trabecular thickness remained significantly lower in the HIVinfected group (Table 2). At the tibia, CSA was similar between groups, but total and 
trabecular vBMD as well as trabecular and cortical thickness were 9-18\% lower in the HIVinfected group, both before and after adjustment for race. In addition, trabecular number was lower, and trabecular separation and network heterogeneity were $15-21 \%$ higher in the HIVinfected group (Table 2, Fig. 2). These measures suggest that the HIV-infected men have thinner, more widely separated, and heterogeneously distributed trabeculae and thinner cortices. There were no significant differences in cortical porosity between the HIV-infected and HIV-uninfected groups. There were no statistically significant differences in HR-pQCT measures between the two HIV-infected groups.

\section{Individual trabeculae segmentation analysis of high-resolution peripheral quantitative computed tomography images}

ITS analysis of the trabecular compartment revealed key microstructural differences in HIVinfected and HIV-uninfected groups at both the radius and tibia (Fig. 3). At the radius, pBV/TV, PR ratio, pTb.N, and pTb.Th, were 31, 30, 8, and 5\% lower in HIV-infected than uninfected controls, respectively, with $\mathrm{pBV} / \mathrm{TV}$ and $\mathrm{pTb}$.Th remaining significant $(P<0.05)$ after adjustment for race/ethnicity. Similarly, at the tibia, pBV/TV, pTb.N, and pTb.Th were 24, 7, and 4\% lower in HIV-infected than uninfected controls, respectively, with significant differences at the $\mathrm{pBV} / \mathrm{TV}, \mathrm{pTb} . \mathrm{N}$, and $\mathrm{pTb}$.Th after adjustment for race/ethnicity. There were no between group differences in rod-specific measures.

The aBV/TV was significantly lower at the radius (-23\%) and tibia (-20\%) in HIV-infected men than uninfected controls. At the radius, $\mathrm{P}-\mathrm{R}$ and $\mathrm{P}-\mathrm{P}$ junction densities were -16 and $-22 \%$, respectively, lower in HIV-infected than controls [27]. At the tibia, only the plateplate junction density was lower, by $17 \%$ in HIV-infected than uninfected men. Most parameters remained significant after adjustment for group differences in race/ethnicity (Fig. 3). Between the men infected perinatally or during adolescence, there was no consistent pattern of microstructural differences at the radius or tibia (data not shown).

\section{Micro finite element analysis of high-resolution peripheral quantitative computed tomography images}

Differences in vBMD and cortical and trabecular microstructure were associated with significant differences in mechanical properties (Fig. 3). Trabecular stiffness was $24 \%$ lower at the radius, and whole bone stiffness was $17 \%$ lower at the radius and $14 \%$ at the tibia. There were no significant differences in men infected perinatally and during adolescence (data not shown).

\section{Discussion}

HIV-infected men in their early 20s, an age when most have achieved peak bone mass, had lower aBMD by DXA at all sites measured, whether infected perinatally or during adolescence. Lower spine, hip, and forearm aBMD could not be accounted for by smaller bone size, as CSA was similar by DXA and HR-pQCTat the radius and tibia. By HR-pQCT, total vBMD was significantly lower at both radius and tibia, predominantly due to marked deficits in the trabecular compartment. Although cortical vBMD did not differ, HIV-infected men had lower cortical area and significantly thinner cortices. Trabecular microarchitecture 
was markedly abnormal at the tibia and radius in HIV-infected men, both by standard HRpQCT analyses, which showed thinner, more widely separated and heterogeneously distributed trabeculae, and by advanced imaging of individual trabeculae by ITS, which revealed fewer and thinner trabecular plates, fewer connections between plates and also between plates and rods. Importantly, these microstructural differences were reflected in lower estimated whole bone and trabecular stiffness (strength) in HIV-infected than uninfected men. The majority of these findings remained significant after adjusting for group differences in race/ethnicity. Our findings suggest that HIV infection and the effects of antiretrovirals early in life are associated with lower peak bone mass, markedly abnormal microarchitecture, and lower estimated bone strength.

In the largest BMD study of HIV-infected children to date, Pediatric AIDS Clinical Trials Group (PACTG) 1045 compared BMC and BMD by DXA in 236 perinatally HIV-infected and 143 HIV-uninfected boys and girls frequency matched by Tanner stage (1-5) and sociodemographic background [3]. In their adjusted models, they found that HIV-infected boys had significantly lower total body BMC and total body and spinal BMD at Tanner 5, lower BMC at Tanner 3-4, and similar BMC and BMD at Tanner 1-2, compared with HIVuninfected boys. Our findings are consistent with the Tanner 5 results from PACTG 1045 and extend their work by demonstrating that lower peak bone mass observed in HIVinfected individuals is accompanied by microarchitectural abnormalities.

The differences we detected between HIV-infected and uninfected young men, between 6 and $19 \%$ lower total and trabecular vBMD, cortical and trabecular thickness, and the higher trabecular separation and network heterogeneity, are similar in magnitude to differences in HR-pQCT parameters observed in studies comparing postmenopausal women with and without low trauma fractures. Such studies report differences in total vBMD and trabecular vBMD ranging from 10 to $20 \%[8,9,25,28,29]$, along with differences in other cortical or trabecular parameters [19,30-32]. Similarly, the magnitude of differences in ITS parameters between HIV groups is comparable to those we documented between postmenopausal women with and without fragility fractures - between 5 and 30\% differences in rod and plate BV/TV, P-R ratio, plate and rod TbN, plate Tb.Th and P-P, P-R, and R-R junction densities [12]. With $\mu \mathrm{FEA}$, bone stiffness was found to be 13-17\% lower in postmenopausal women with ankle fractures compared with those with no fractures [19]. Therefore, these results suggest that men infected with HIV perinatally or during adolescence have lower bone strength and thus may be at higher risk of fracture than their peers at peak bone mass. With aging, both the cortical and trabecular deficits in these young men can be expected to progress further [33], placing them at even higher risk of fracture than uninfected men and perhaps also men infected with HIV later in life.

Notably, there were few differences in bone mass and structure between HIV-infected groups. Because men infected with HIV perinatally have had longer exposure to the HIV virus and to antiretroviral drugs, we had hypothesized that peak bone mass would be lower and HR-pQCT abnormalities more pronounced in perinatally infected men than those infected during adolescence. The perinatally infected group appeared to have smaller bone size as well as decreased cortical thickness and vBMD than the group infected during adolescence, but these differences did not reach statistical significance in this small study. 
Mean $\mathrm{CD}^{+}$cell and HIV-1 RNA levels at the time of evaluation did not differ between groups nor did they correlate consistently with either aBMD or vBMD (data not shown). Tenofovir is the antiretroviral with the clearest association with bone loss both at the initiation and switch phases of treatment [34,35]. Therefore, the fact that a greater proportion of the group infected during adolescence was taking tenofovir than the perinatally infected group (93 versus $67 \%, P=0.24$ ) with longer duration of exposure (3.7 \pm 3.2 versus $1.4 \pm 0.8$ years, $P=0.05$ ) may partially explain why group differences were smaller than expected. In addition, there may be other unmeasured exposures among the group infected during adolescence that impact bone. A recent preexposure prophylaxis study found that a significant proportion of men who have sex with men at risk of HIV acquisition had low BMD at baseline that was associated with amphetamine and inhalant use [36]. It is also possible that bone mass acquisition may be particularly vulnerable to the impact of HIV infection and ART treatment during the skeletal growth phase of adolescence. A larger, longitudinal study is necessary to confirm these results.

To our knowledge, this is the first study to examine peak bone mass and bone microarchitecture with HR-pQCT in HIV-infected individuals. A major strength of our study is that the control group was similar in age, height, weight, and CSA of spine, hip, and radius; however, it remains possible that skeletal maturation is delayed and peak bone mass is acquired later in HIV-infected individuals.

This study also has several limitations. The sample size was small and may have limited our ability to detect differences between HIV-infected who contracted the disease at birth and those who contracted it during adolescence. Cortical area at the radius was approximately $15 \%$ lower in men infected perinatally than in adolescence; however, with our sample size, we had only $39 \%$ power to detect that difference. The sample size also limited our ability to determine the impact of exposure to tenofovir or other antiretrovirals on BMD. Additionally, we do not have data on important covariates such as vitamin D levels, diet, and exercise, which may have attenuated expected differences in BMD. Another limitation of the study is the difference in race/ethnicity between the groups. Age at which BMD acquisition reaches a plateau phase is similar across race and ethnicity, but black men attain higher mean aBMD values at the hip and spine than nonblack (Asian, Hispanic, and whites) male youth [37]. There may also be microarchitectural differences between African-Americans and Hispanics, but data are limited. Additionally, the majority of our Hispanics were of Caribbean origin. There is a great deal of racial admixture in these Hispanic populations, and aBMD and HR-pQCT characteristics of Hispanics of Caribbean origin have not been characterized. As there were more African-Americans than Hispanics in the HIV-infected group, and aBMD is likely to be greater in African-Americans than Hispanics, the race/ ethnicity imbalance of our sample would have biased our findings toward the null. Finally, the differences in HR-pQCT parameters remained significant after adjustment for race/ ethnicity. Finally, there are some technical limitations due to the resolution when measuring Ct.Po and performing ITS; however, these methods have been validated against higher resolution technologies [11,21].

Recent data from several large cohort studies suggest that incidence of fragility and nonfragility fractures are 1.2-2.4 times greater in HIV-infected than uninfected adults [38- 
40]. Although the largest difference in fracture rates occur among older individuals [41], higher fracture rates have also been reported in younger HIV-infected individuals $[39,40,42]$. Our data suggest that men infected with HIV early in life have lower peak bone mass, a thinner cortical shell, markedly abnormal trabecular bone microstructure with deficiencies in trabecular plates and axial bone volume fraction, and reduced bone strength. These deficits may place them at higher risk of fractures as they age than uninfected individuals and HIV-infected individuals who were infected later in life, after acquisition of peak bone mass. With more than 3 million children under age 15 and nearly 5 million young adults, age 15-24 living with HIV worldwide [43], bone health of those infected with HIV early in life is an area of concern and warrants further study.

\section{Acknowledgments}

The authors would like to thank the participants and Meryl Ueno.

M.T.Y. and S.M.A contributed to study design. E.B.L., J.S., J.N., M.F., and D.L.B contributed to study conduct. J.S. contributed to data collection. C.A.Z., K.K.N., and B.Z. contributed to data analysis. M.T.Y., S.M.A., E.S., and X.E.G. contributed to data interpretation. M.T.Y. and S.M.A. drafted the article. E.S., N.N., K.K.N., M.F., and X.E.G. revised the article content. M.T.Y. and S.M.A. approved final version of article.

This work was supported in part by National Institute of Health Grants R01 AI095089 (M.T.Y.) and R01 HD073977 (S.M.A. and M.T.Y.). The content is solely the responsibility of the authors and does not necessarily represent the official views of the NIH. M.T.Y. has served as a consultant for Gilead and Abbott.

\section{References}

1. Heaney RP, Abrams S, Dawson-Hughes B, Looker A, Marcus R, Matkovic V, et al. Peak bone mass. Osteoporos Int. 2000; 11:985-1009. [PubMed: 11256898]

2. Jacobson DL, Spiegelman D, Duggan C, Weinberg GA, Bechard L, Furuta L, et al. Predictors of bone mineral density in human immunodeficiency virus-1 infected children. J Pediatr Gastroenterol Nutr. 2005; 41:339-346. [PubMed: 16131991]

3. Jacobson DL, Lindsey JC, Gordon CM, Moye J, Hardin DS, Mulligan K, et al. Total body and spinal bone mineral density across Tanner stage in perinatally HIV-infected and uninfected children and youth in PACTG 1045. AIDS. 2010; 24:687-696. [PubMed: 20168204]

4. Arpadi SM, Horlick M, Thornton J, Cuff PA, Wang J, Kotler DP. Bone mineral content is lower in prepubertal HIV-infected children. J Acquir Immune Defic Syndr. 2002; 29:450-454. [PubMed: 11981360]

5. O’Brien KO, Razavi M, Henderson RA, Caballero B, Ellis KJ. Bone mineral content in girls perinatally infected with HIV. Am J Clin Nutr. 2001; 73:821-826. [PubMed: 11273859]

6. Mulligan K, Harris DR, Emmanuel P, Fielding RA, Worrell C, Kapogiannis BG, et al. Low bone mass in behaviorally HIV-infected young men on antiretroviral therapy: Adolescent Trials Network Study 021B. Clin Infect Dis. 2012; 55:461-468. [PubMed: 22573848]

7. Pitukcheewanont P, Safani D, Church J, Gilsanz V. Bone measures in HIV-1 infected children and adolescents: disparity between quantitative computed tomography and dual-energy X-ray absorptiometry measurements. Osteoporos Int. 2005; 16:1393-1396. [PubMed: 15702261]

8. Sornay-Rendu E, Boutroy S, Munoz F, Delmas PD. Alterations of cortical and trabecular architecture are associated with fractures in postmenopausal women, partially independent of decreased BMD measured by DXA: the OFELY study. J Bone Miner Res. 2007; 22:425-433. [PubMed: 17181395]

9. Boutroy S, Bouxsein ML, Munoz F, Delmas PD. In vivo assessment of trabecular bone microarchitecture by high-resolution peripheral quantitative computed tomography. J Clin Endocrinol Metab. 2005; 90:6508-6515. [PubMed: 16189253] 
10. Burghardt AJ, Kazakia GJ, Majumdar S. A local adaptive threshold strategy for high resolution peripheral quantitative computed tomography of trabecular bone. Ann Biomed Eng. 2007; 35:1678-1686. [PubMed: 17602299]

11. Liu XS, Shane E, McMahon DJ, Guo XE. Individual trabecula segmentation (ITS)-based morphological analysis of microscale images of human tibial trabecular bone at limited spatial resolution. J Bone Miner Res. 2011; 26:2184-2193. [PubMed: 21557311]

12. Liu XS, Stein EM, Zhou B, Zhang CA, Nickolas TL, Cohen A, et al. Individual trabecula segmentation (ITS)-based morphological analyses and microfinite element analysis of HR-pQCT images discriminate postmenopausal fragility fractures independent of DXA measurements. J Bone Miner Res. 2012; 27:263-272. [PubMed: 22072446]

13. Liu XS, Sajda P, Saha PK, Wehrli FW, Bevill G, Keaveny TM, et al. Complete volumetric decomposition of individual trabecular plates and rods and its morphological correlations with anisotropic elastic moduli in human trabecular bone. J Bone Miner Res. 2008; 23:223-235. [PubMed: 17907921]

14. Ostertag A, Collet C, Chappard C, Fernandez S, Vicaut E, Cohen-Solal M, et al. A case-control study of fractures in men with idiopathic osteoporosis: fractures are associated with older age and low cortical bone density. Bone. 2013; 52:48-55. [PubMed: 23010106]

15. Stephen MD, Bryant WP, Wilson DP. Self-assessment of sexual maturation in children and adolescents with diabetes mellitus. Endocr Pract. 2008; 14:840-845. [PubMed: 18996811]

16. Cohen A, Liu XS, Stein EM, McMahon DJ, Rogers HF, Lemaster J, et al. Bone microarchitecture and stiffness in premenopausal women with idiopathic osteoporosis. J Clin Endocrinol Metab. 2009; 94:4351-4360. [PubMed: 19837923]

17. Laib A, Hauselmann HJ, Ruegsegger P. In vivo high resolution 3D-QCT of the human forearm. Technol Healthcare. 1998; 6:329-337.

18. Liu XS, Zhang XH, Sekhon KK, Adams MF, McMahon DJ, Bilezikian JP, et al. High-resolution peripheral quantitative computed tomography can assess microstructural and mechanical properties of human distal tibial bone. J Bone Miner Res. 2010; 25:746-756. [PubMed: 19775199]

19. Stein EM, Liu XS, Nickolas TL, Cohen A, Thomas V, McMahon DJ, et al. Abnormal microarchitecture and reduced stiffness at the radius and tibia in postmenopausal women with fractures. J Bone Miner Res. 2010; 25:2572-2581. [PubMed: 20564238]

20. Buie HR, Campbell GM, Klinck RJ, MacNeil JA, Boyd SK. Automatic segmentation of cortical and trabecular compartments based on a dual threshold technique for in vivo micro-CT bone analysis. Bone. 2007; 41:505-515. [PubMed: 17693147]

21. Nishiyama KK, Macdonald HM, Buie HR, Hanley DA, Boyd SK. Postmenopausal women with osteopenia have higher cortical porosity and thinner cortices at the distal radius and tibia than women with normal aBMD: an in vivo HR-pQCT study. J Bone Miner Res. 2010; 25:882-890. [PubMed: 19839766]

22. Burghardt AJ, Kazakia GJ, Ramachandran S, Link TM, Majumdar S. Age- and gender-related differences in the geometric properties and biomechanical significance of intracortical porosity in the distal radius and tibia. J Bone Miner Res. 2010; 25:983-993. [PubMed: 19888900]

23. Liu XS, Sajda P, Saha PK, Wehrli FW, Guo XE. Quantification of the roles of trabecular microarchitecture and trabecular type in determining the elastic modulus of human trabecular bone. J Bone Miner Res. 2006; 21:1608-1617. [PubMed: 16995816]

24. Vilayphiou N, Boutroy S, Sornay-Rendu E, Van Rietbergen B, Munoz F, Delmas PD, et al. Finite element analysis performed on radius and tibia HR-pQCT images and fragility fractures at all sites in postmenopausal women. Bone. 2010; 46:1030-1037. [PubMed: 20044044]

25. Melton LJ 3rd, Christen D, Riggs BL, Achenbach SJ, Muller R, van Lenthe GH, et al. Assessing forearm fracture risk in postmenopausal women. Osteoporos Int. 2010; 21:1161-1169. [PubMed: 19714390]

26. Hollister SJ, Brennan JM, Kikuchi N. A homogenization sampling procedure for calculating trabecular bone effective stiffness and tissue level stress. J Biomech. 1994; 27:433-444. [PubMed: 8188724]

27. Liu XS, Cohen A, Shane E, Stein E, Rogers H, Kokolus SL, et al. Individual trabeculae segmentation (ITS)-based morphological analysis of high-resolution peripheral quantitative 
computed tomography images detects abnormal trabecular plate and rod microarchitecture in premenopausal women with idiopathic osteoporosis. J Bone Miner Res. 2010; 25:1496-1505. [PubMed: 20200967]

28. Melton LJ 3rd, Riggs BL, Keaveny TM, Achenbach SJ, Hoffmann PF, Camp JJ, et al. Structural determinants of vertebral fracture risk. J Bone Miner Res. 2007; 22:1885-1892. [PubMed: 17680721]

29. Vico L, Zouch M, Amirouche A, Frere D, Laroche N, Koller B, et al. High-resolution pQCT analysis at the distal radius and tibia discriminates patients with recent wrist and femoral neck fractures. J Bone Miner Res. 2008; 23:1741-1750. [PubMed: 18665795]

30. Cohen A, Dempster DW, Muller R, Guo XE, Nickolas TL, Liu XS, et al. Assessment of trabecular and cortical architecture and mechanical competence of bone by high-resolution peripheral computed tomography: comparison with transiliac bone biopsy. Osteoporos Int. 2010; 21:263273. [PubMed: 19455271]

31. Boutroy S, Van Rietbergen B, Sornay-Rendu E, Munoz F, Bouxsein ML, Delmas PD. Finite element analysis based on in vivo HR-pQCT images of the distal radius is associated with wrist fracture in postmenopausal women. J Bone Miner Res. 2008; 23:392-399. [PubMed: 17997712]

32. Sornay-Rendu E, Cabrera-Bravo JL, Boutroy S, Munoz F, Delmas PD. Severity of vertebral fractures is associated with alterations of cortical architecture in postmenopausal women. J Bone Miner Res. 2009; 24:737-743. [PubMed: 19113929]

33. Riggs BL, Melton LJ, Robb RA, Camp JJ, Atkinson EJ, McDaniel L, et al. A population-based assessment of rates of bone loss at multiple skeletal sites: evidence for substantial trabecular bone loss in young adult women and men. J Bone Miner Res. 2008; 23:205-214. [PubMed: 17937534]

34. McComsey GA, Kitch D, Daar ES, Tierney C, Jahed NC, Tebas P, et al. Bone mineral density and fractures in antiretroviral-naive persons randomized to receive abacavir-lamivudine or tenofovir disoproxil fumarate-emtricitabine along with efavirenz or atazanavir-ritonavir: Aids Clinical Trials Group A5224s, a substudy of ACTG A5202. J Infect Dis. 2011; 203:1791-1801. [PubMed: 21606537]

35. Haskelberg H, Hoy JF, Amin J, Ebeling PR, Emery S, Carr A, et al. Changes in bone turnover and bone loss in HIV-infected patients changing treatment to tenofovir-emtricitabine or abacavirlamivudine. PLoS One. 2012; 7:e38377. [PubMed: 22719882]

36. Liu AY, Vittinghoff E, Sellmeyer DE, Irvin R, Mulligan K, Mayer K, et al. Bone mineral density in HIV-negative men participating in a tenofovir preexposure prophylaxis randomized clinical trial in San Francisco. PLoS One. 2011; 6:e23688. [PubMed: 21897852]

37. Bachrach LK, Hastie T, Wang MC, Narasimhan B, Marcus R. Bone mineral acquisition in healthy Asian, Hispanic, black, and Caucasian youth: a longitudinal study. J Clin Endocrinol Metab. 1999; 84:4702-4712. [PubMed: 10599739]

38. Arnsten JH, Freeman R, Howard AA, Floris-Moore M, Lo Y, Klein RS. Decreased bone mineral density and increased fracture risk in aging men with or at risk for HIV infection. AIDS. 2007; 21:617-623. [PubMed: 17314524]

39. Hansen AB, Gerstoft J, Kronborg G, Larsen CS, Pedersen C, Pedersen G, et al. Incidence of low and high-energy fractures in persons with and without HIV infection: a Danish population-based cohort study. AIDS. 2012; 26:285-293. [PubMed: 22095195]

40. Young B, Dao CN, Buchacz K, Baker R, Brooks JT, Investigators HIVOS. Increased rates of bone fracture among HIV-infected persons in the HIV Outpatient Study (HOPS) compared with the US general population, 2000-2006. Clin Infect Dis. 2011; 52:1061-1068. [PubMed: 21398272]

41. Guerri-Fernandez R, Vestergaard P, Carbonell C, Knobel H, Aviles FF, Soria Castro A, et al. HIV infection is strongly associated with hip fracture risk, independently of age, gender and comorbidities: a population-based cohort study. J Bone Miner Res. 2013; 28:1259-1263. [PubMed: 23362011]

42. Shiau S, Lund E, Arpadi SM, Yin MT. Incident fractures in HIV-infected individuals: a systematic review and meta-analysis. AIDS. 2013 in press.

43. UNICEF. Opportunity in crisis: preventing HIV from early adolescence to young adulthood. United Nations publication: 2011. 


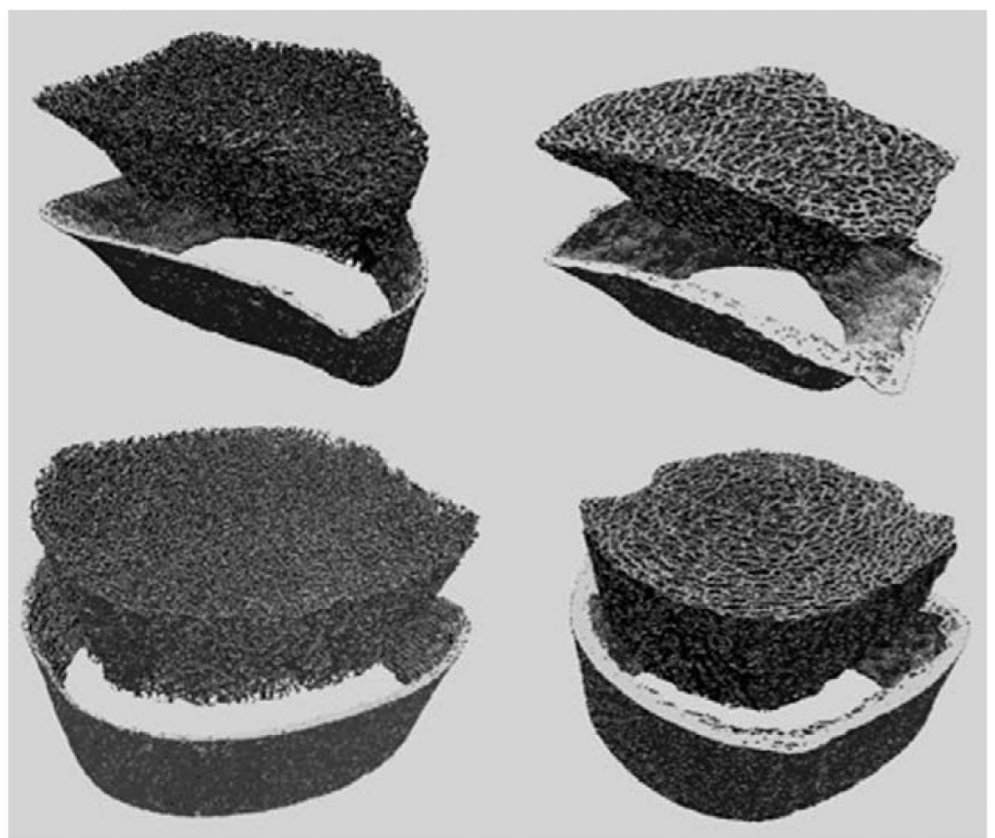

Fig. 1. Representative high-resolution peripheral quantitative computed tomography (HRpQCT) images from 24-year-old HIV-infected (left) and HIV-uninfected (right) AfricanAmerican men at the radius (top) and tibia (bottom)

HR-pQCT, high-resolution peripheral quantitative computed tomography. 


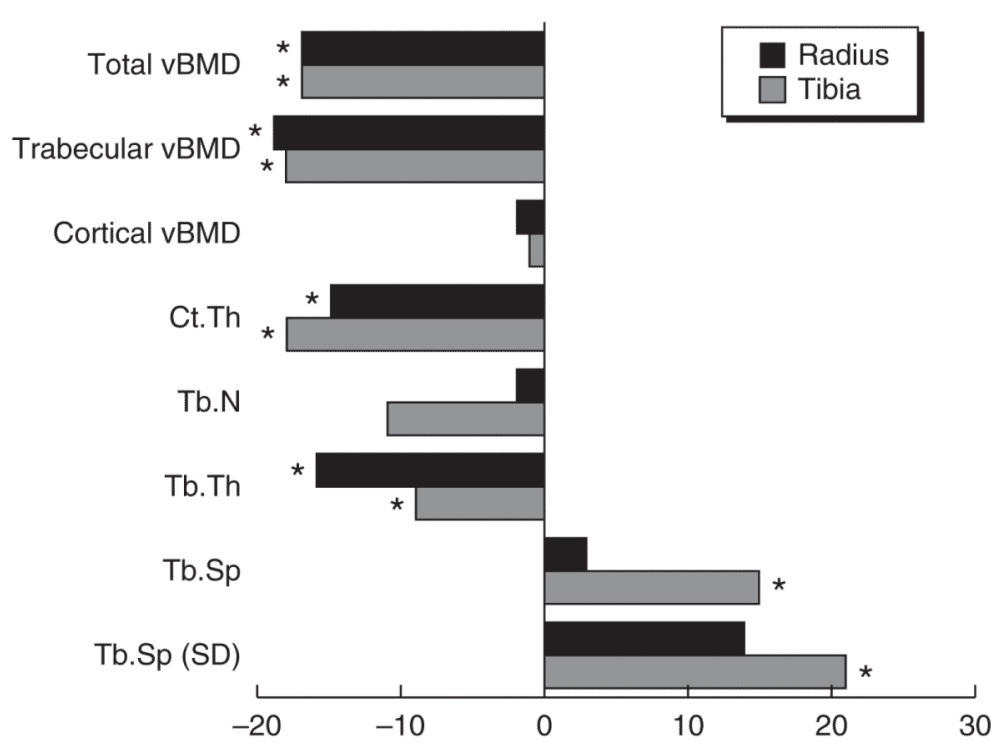

Fig. 2. Percentage difference in high-resolution peripheral quantitative computed tomography measures between HIV-infected and HIV-uninfected men at the radius and tibia

Ct.Th, cortical thickness; HR-pQCT, high-resolution peripheral quantitative computed tomography; Tb.N, trabecular number; Tb.Sp (SD), trabecular separation standard deviation; Tb.SP, trabecular separation; vBMD, volumetric bone mineral density. $* P<0.05$ after adjustment for race/ethnicity. 


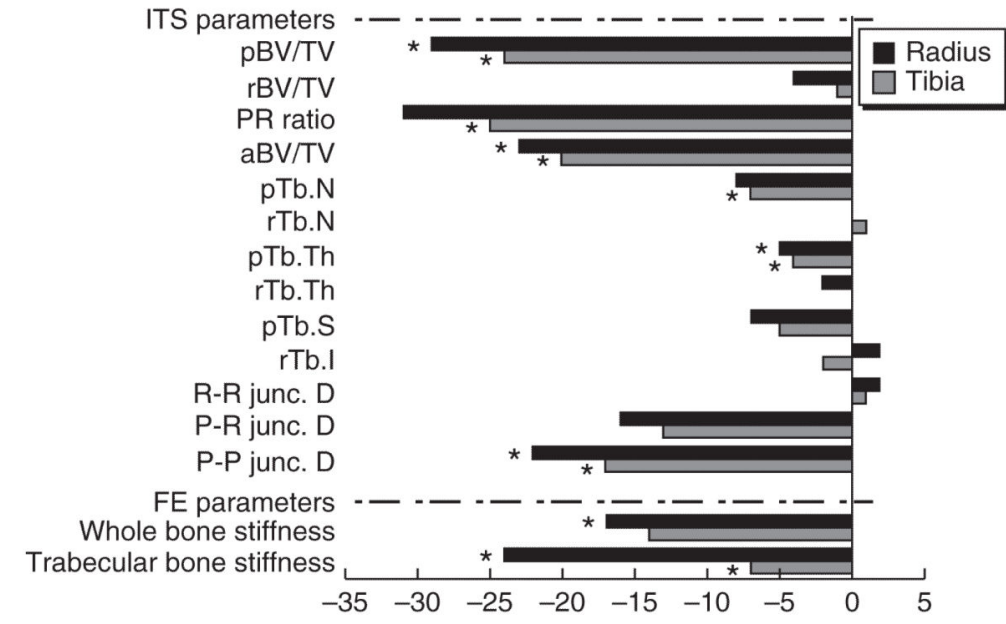

Fig. 3. Percentage difference in individual trabeculae segmentation and micro finite element analysis measures between HIV-infected and HIV-uninfected men at the radius and tibia $\mu F E A$, micro finite element analysis; ITS, individual trabeculae segmentation; $\mathrm{pBV} / \mathrm{TV}$ and $\mathrm{rBV} / \mathrm{TV}$, plate and rod bone volume fraction; P-P, P-R, and R-R Junc.D, plate-plate, platerod, and rod-rod junction density; pTb.N and rTb.N, plate and rod number; P-R ratio, plateto-rod ratio; pTb.Th and rTb.Th, plate and rod thickness; pTb.S, plate surface area; rTb. $\ell$ mm, rod length. ${ }^{*} P<0.05$ after adjustment for race/ethnicity. 
Table 1

Characteristics and dual X-ray absorptiometry results of young men infected with HIV early in life and uninfected controls

\begin{tabular}{|c|c|c|c|}
\hline & HIV-infected $(N=30)$ & Uninfected $(N=15)$ & $P$ value \\
\hline \multicolumn{4}{|l|}{ Patient characteristics } \\
\hline Age & $22.5 \pm 0.3$ & $22.0 \pm 0.5$ & 0.31 \\
\hline Race/ethnicity (African-American, Hispanic) & $60 \% \mathrm{AA} ; 40 \% \mathrm{H}$ & $20 \%$ AA; $80 \% \mathrm{H}$ & 0.01 \\
\hline Height (cm) & $173 \pm 2$ & $173 \pm 2$ & 0.97 \\
\hline Weight (kg) & $77 \pm 4$ & $75 \pm 5$ & 0.72 \\
\hline BMI $\left(\mathrm{kg} / \mathrm{cm}^{2}\right)$ & $26.0 \pm 0.6$ & $24,8 \pm 1.3$ & 0.71 \\
\hline Current smoking (\%) & $43 \%$ & $20 \%$ & 0.26 \\
\hline Alcohol use $>2$ drinks/day $(\%)$ & $3 \%$ & $0 \%$ & 0.49 \\
\hline \multicolumn{4}{|l|}{ DXA cross-sectional area and body composition } \\
\hline $\operatorname{LSCSA}\left(\mathrm{cm}^{2}\right)$ & $63.5 \pm 1.1$ & $64.9 \pm 2.8$ & 0.45 \\
\hline FN CSA $\left(\mathrm{cm}^{2}\right)$ & $5.4 \pm 0.1$ & $5.2 \pm 0.1$ & 0.34 \\
\hline TH CSA $\left(\mathrm{cm}^{2}\right)$ & $39.7 \pm 0.7$ & $40.1 \pm 1.5$ & 0.78 \\
\hline $\mathrm{R} 13 \mathrm{CSA}\left(\mathrm{cm}^{2}\right)$ & $3.0 \pm 0.1$ & $2.9 \pm 0.1$ & 0.41 \\
\hline $\operatorname{UDR}$ CSA $\left(\mathrm{cm}^{2}\right)$ & $3.8 \pm 0.9$ & $3.8 \pm 1.0$ & 0.67 \\
\hline Total body fat (\%) & $22.3 \pm 1.1$ & $21.3 \pm 1.9$ & 0.61 \\
\hline Trunk fat $(\%)$ & $21.3 \pm 1.4$ & $19.8 \pm 2.2$ & 0.59 \\
\hline \multicolumn{4}{|l|}{ DXA bone mineral content and density } \\
\hline LS aBMD $\left(\mathrm{g} / \mathrm{cm}^{2}\right)$ & $1.04 \pm 0.02$ & $1.11 \pm 0.03$ & 0.049 \\
\hline $\mathrm{FN}$ aBMD $\left(\mathrm{g} / \mathrm{cm}^{2}\right)$ & $0.97 \pm 0.03$ & $1.06 \pm 0.04$ & 0.07 \\
\hline TH aBMD $\left(\mathrm{g} / \mathrm{cm}^{2}\right)$ & $1.07 \pm 0.03$ & $1.15 \pm 0.04$ & 0.09 \\
\hline R13 aBMD $\left(\mathrm{g} / \mathrm{cm}^{2}\right)$ & $0.75 \pm 0.01$ & $0.77 \pm 0.02$ & 0.01 \\
\hline UDR aBMD $\left(\mathrm{g} / \mathrm{cm}^{2}\right)$ & $0.50 \pm 0.01$ & $0.56 \pm 0.01$ & 0.002 \\
\hline LS Z-score & $-1.01 \pm 0.23$ & $0.01 \pm 0.27$ & $<0.01$ \\
\hline FN Z-score & $-0.37 \pm 0.19$ & $0.50 \pm 0.25$ & 0.01 \\
\hline TH Z-score & $-0.22 \pm 0.20$ & $0.62 \pm 0.25$ & 0.01 \\
\hline R13 Z-score & $-1.27 \pm 0.25$ & $-0.05 \pm 0.32$ & 0.007 \\
\hline UDR Z-score & $-0.70 \pm 0.18$ & $0.24 \pm 0.21$ & 0.002 \\
\hline
\end{tabular}

AA, African-American; H, Hispanic; aBMD, areal bone mineral density; BMC, bone mineral content; CSA, cross-sectional area; DR, 1/3 distal radius; DXA, dual X-ray absorptiometry; FN, femoral neck; LS, lumbar spine; TH, total hip; UDR, ultradistal radius. 
Table 2

High-resolution peripheral quantitative computed tomography measures in young men infected with HIV early in life and uninfected controls (mean \pm SEM)

\begin{tabular}{|c|c|c|c|c|c|c|}
\hline & $\begin{array}{l}\text { HIV-infected } \\
\quad(N=30)\end{array}$ & $\begin{array}{l}\text { Uninfected } \\
(N=15)\end{array}$ & $P$ value & $\begin{array}{l}\text { Perinatal infection } \\
\quad(N=15)\end{array}$ & $\begin{array}{c}\text { Adolescent infection } \\
\qquad(N=15)\end{array}$ & $P$ value \\
\hline \multicolumn{7}{|l|}{ Radius } \\
\hline Total area $\left(\mathrm{mm}^{2}\right)$ & $344 \pm 14$ & $328 \pm 23$ & 0.32 & $337 \pm 20$ & $351 \pm 20$ & 0.59 \\
\hline Ct. area $\left(\mathrm{mm}^{2}\right)$ & $63.4 \pm 2.8$ & $73.7 \pm 3.3$ & 0.03 & $58.7 \pm 4.1$ & $68.1 \pm 3.7$ & 0.10 \\
\hline $\mathrm{Tb}$. area $\mathrm{mm}^{2}$ ) & $281 \pm 14$ & $255 \pm 26$ & 0.32 & $278 \pm 22$ & $283 \pm 18$ & 0.85 \\
\hline Total vBMD $\left(\mathrm{mgHA} / \mathrm{cm}^{3}\right)$ & $324 \pm 11$ & $388 \pm 15$ & $0.002^{*}$ & $318 \pm 21$ & $330 \pm 10$ & 0.61 \\
\hline Ct. vBMD $\left(\mathrm{mgHA} / \mathrm{cm}^{3}\right)$ & $814 \pm 13$ & $829 \pm 15$ & 0.49 & $796 \pm 20$ & $831 \pm 15$ & 0.17 \\
\hline Tb. vBMD $\left(\mathrm{mg} \mathrm{HA} / \mathrm{cm}^{3}\right)$ & $188 \pm 6$ & $233 \pm 7$ & $>0.0001^{*}$ & $187 \pm 9$ & $189 \pm 9$ & 0.85 \\
\hline Ct. thickness $(\mu \mathrm{m})$ & $992 \pm 37$ & $1170 \pm 53$ & $0.006^{*}$ & $955 \pm 64$ & $1027 \pm 36$ & 0.32 \\
\hline Ct. porosity (\%) & $6.5 \pm 0.4$ & $6.7 \pm 0.5$ & 0.77 & $6.7 \pm 0.6$ & $6.4 \pm 0.4$ & 0.68 \\
\hline Tb. thickness $(\mu \mathrm{m})$ & $75 \pm 2$ & $88 \pm 4$ & $0.004^{*}$ & $72 \pm 3$ & $78 \pm 4$ & 0.22 \\
\hline Tb. number $(1 / \mathrm{mm})$ & $2.0 \pm 0.04$ & $2.1 \pm 0.1$ & 0.12 & $2.2 \pm 0.1$ & $2.0 \pm 0.1$ & 0.19 \\
\hline Tb. separation $(\mu \mathrm{m})$ & $408 \pm 11$ & $370 \pm 18$ & 0.052 & $396 \pm 14$ & $420 \pm 16$ & 0.27 \\
\hline Tb. separation SD $(\mu \mathrm{m})$ & $166 \pm 6$ & $146 \pm 10$ & 0.10 & $159 \pm 7$ & $173 \pm 9$ & 0.23 \\
\hline \multicolumn{7}{|l|}{ Tibia } \\
\hline Total area $\left(\mathrm{mm}^{2}\right)$ & $811 \pm 27$ & $775 \pm 45$ & 0.22 & $787 \pm 40$ & $834 \pm 38$ & 0.40 \\
\hline Ct. area $\left(\mathrm{mm}^{2}\right)$ & $149 \pm 5$ & $176 \pm 9$ & $0.007^{*}$ & $147 \pm 8$ & $152 \pm 6$ & 0.63 \\
\hline Tb. area $\mathrm{mm}^{2}$ ) & $661 \pm 27$ & $599 \pm 45$ & 0.22 & $640 \pm 40$ & $683 \pm 38$ & 0.45 \\
\hline Total vBMD $\left(\mathrm{mgHA} / \mathrm{cm}^{3}\right)$ & $320 \pm 10$ & $386 \pm 15$ & $0.0004^{*}$ & $320 \pm 14$ & $320 \pm 14$ & 0.98 \\
\hline Ct. vBMD $\left(\mathrm{mgHA} / \mathrm{cm}^{3}\right)$ & $876 \pm 8$ & $880 \pm 11$ & 0.79 & $863 \pm 11$ & $888 \pm 10$ & 0.10 \\
\hline Tb. $\operatorname{vBMD}\left(\mathrm{mg} \mathrm{HA} / \mathrm{cm}^{3}\right)$ & $186 \pm 6$ & $230 \pm 10$ & $0.0003^{*}$ & $187 \pm 8$ & $186 \pm 9$ & 0.93 \\
\hline Ct. thickness $(\mu \mathrm{m})$ & $1483 \pm 45$ & $1803 \pm 120$ & $0.02^{*}$ & $1462 \pm 70$ & $1503 \pm 57$ & 0.66 \\
\hline Ct. porosity (\%) & $5.9 \pm 0.3$ & $7.4 \pm 0.7$ & 0.58 & $6.1 \pm 0.4$ & $5.7 \pm 0.3$ & 0.40 \\
\hline Tb. thickness $(\mu \mathrm{m})$ & $80 \pm 2$ & $88 \pm 9$ & $0.052^{*}$ & $80 \pm 3$ & $80 \pm 4$ & 0.95 \\
\hline Tb. number $(1 / \mathrm{mm})$ & $2.0 \pm 0.1$ & $2.2 \pm 0.1$ & 0.02 & $2.0 \pm 0.1$ & $2.0 \pm 0.1$ & 0.82 \\
\hline Tb. separation $(\mu \mathrm{m})$ & $438 \pm 13$ & $380 \pm 24$ & $0.02^{*}$ & $434 \pm 16$ & $442 \pm 21$ & 0.77 \\
\hline Tb. separation $\mathrm{SD}(\mu \mathrm{m})$ & $202 \pm 9$ & $166 \pm 14$ & $0.03^{*}$ & $198 \pm 10$ & $206 \pm 16$ & 0.69 \\
\hline
\end{tabular}

$\mathrm{Ct}$, cortical;HR-pQCT, high-resolution peripheral quantitative computed tomography;Tb, trabecular;vBMD, volumetric bone mineral density. Ct. thickness, vBMD, and porosity were calculated using the autosegmentation method described in methods.

${ }^{*}<0.05$ after adjustment for race/ethnicity. 